Gazi University
Journal of Science
http://dergipark.gov.tr/gujs

\title{
Classification of 8-dimensional Nilsolitons by Symbolic Computation
}

\author{
Hulya KADIOGLU \\ Department of Mathematics and Science Education, Yildiz Technical University, Istanbul, Turkey \\ Highlights \\ - Create algorithms for the classification of 8-dimensional nilsoliton metrics on Lie algebras. \\ - Use symbolic computation in the classification process. \\ - Classifications by rank, index, nilpotency, derivation type, structure constants.
}

\begin{tabular}{l} 
Article Info \\
\hline \\
Received: 13 Apr 2021 \\
Accepted: 13 Sep 2021 \\
Keywords \\
\hline Solvable Lie algebras \\
Symbolic computation \\
Nilpotent Lie groups \\
Solvable Lie groups
\end{tabular}

\section{INTRODUCTION}

Symbolic computation is an area of mathematics that deals with developing, executing and applying the algorithms to manipulate and analyze the mathematical expressions or other mathematical objects. It concerns with the formulation of algorithms and mostly exact solutions of symbolic mathematical problems. Implementing these algorithms with regards to the control structures or operations that are available in computer programming environments like FORTRAN, GAP, MATLAB ETC is also another part of the symbolic computation process. It is a useful tool since it does the computations more productively and accurately than doing by hand, or does the computations that are almost impossible to carry out by hand.

In recent years, symbolic computation methods have been used for Lie algebra theory which is mostly result highly complex symbolic expressions that are very difficult to carry out without the aid of computer algorithms. Therefore, it becomes possible to work with structural objects in the Lie algebra theory by the introduction and implementation of new algorithms. Nowadays, computer algorithms help computing most of the basic objects in structure theory of Lie algebras, such as construction of quotient algebras, minimal matrix representation, the centralizer or normalizer of a Lie algebra [1-5].

There are three methods to represent a Lie algebra and its related structures: Representing a Lie algebra as a sub algebra of $g l(n)$, using table of its structure constants or using generators and relations [3]. In this paper, we use the table of structure constants related to a Lie algebra. We vary the Lie algebra structure by finding structure constants. Namely we determine a Lie algebra $\eta$ with a fixed basis $\left\{X_{i}: 1 \leq i \leq n\right\}$ 
explicitly by given multiplication table, consisting of structure constants $\alpha_{i j}^{k}$ which are defined by the relations

$$
\left[X_{i}, X_{j}\right]=\alpha_{i j}^{k} X_{k}
$$

For the use of symbolic computations, we use the index set

$\Lambda=\left\{(i . j . k): \alpha_{i j}^{k} \neq 0, i<j<k\right\}$

to encode nonzero structure constants. Since we work on nilpotent Lie algebras, it is possible to consider $i<j<k$ condition because of the skew-symmetry, and nilpotency. To construct such index set, we use triples $(i . j . k) \in \Lambda$ such that $\mathrm{i}<\mathrm{j}<\mathrm{k}$, and if $(i . j . k),(i . j . m) \in \Lambda$ then $\mathrm{k}=\mathrm{m}$. Also, if $\left(i . j_{1} \cdot k\right),\left(i . j_{2} . k\right) \in$ $\Lambda$ then $j_{1}=j_{2}$. Basically, we fix a basis $\left\{X_{i}: 1 \leq i \leq n\right\}$ for the nilpotent Lie algebra $\eta$ with $\left[X_{i}, X_{j}\right]=$ $\alpha_{i j}^{k} X_{k} \neq 0$. Here for every $i, j$, there is a unique $k$ such that $\alpha_{i j}^{k} \neq 0$ and for every $i, k$, there is a unique $\mathrm{j}$, such that $\alpha_{i j}^{k} \neq 0$. The existence of such basis was proven by Nikolayevsky in [6].

It is well known that one can define several different Riemannian metrics on Lie groups. Among them the most preferable is Einstein metrics mainly because of its Ricci tensor complying the Einstein metric:

$R i c=c g$ for some constant $c \in I R$. But unfortunately, Einstein metrics cannot be defined on non-abelian nilpotent Lie algebras. Therefore, we consider a left invariant metric $g$ on a nilpotent Lie group $\mathrm{G}$ called nilsoliton metrics satisfying the weaker condition

$\operatorname{Ric}_{g}=\beta I+D$

For some $\beta \epsilon I R$ and $D \in \operatorname{Der}(\eta)$. Here Ric $g$ denotes the Ricci operator of $\eta$, where $\eta$ is the Lie algebra of $\mathrm{G}$ and $\operatorname{Der}(\eta)$ denotes the Lie algebra of derivations of $\eta$. Equation (2) is called nilsoliton condition, $\mathrm{D}$ is called nilsoliton derivation, and $\beta$ is called nilsoliton constant.

Nilsolitons are an important topic in mathematics for several reasons. First, nilsoliton metric Lie algebras are unique up to isometry and scaling. In [7], Theorem 2.11 states that a nilpotent Lie algebra admits a nilsoliton metric if and only if it is an Einstein nilradical. Therefore, it indicates that classification of nilsoliton metrics on a nilpotent Lie algebra is equivalent to the same of Einstein nilradicals. On the other hand, an Einstein solvmanifold $\delta$ can completely be determined by the Lie algebra $\eta=[\beta, \beta]$. Therefore, the study of solvmanifolds are actually the study of nilsolitons. See $[7,8]$ for a survey on nilsoliton metric Lie algebras.

Nilsoliton metrics are classified in different dimensions by several approaches [6, 7, 9-14]. In dimension 7 and 8 with non-singular Gram matrix in [15] and in dimension 7 in [10, 16]. Also, in [17] the ordered type of nilsoliton metrics in higher dimensions were classified. In this paper, we classify 8-dimensinal nilsoliton metric Lie algebras with simple nilsoliton derivation D corresponding to a singular Gram matrix. On the other hand, Theorem 7 in [18] suggests that the direct sum of nilsolitons is again a nilsoliton. In dimension 7 and lower, nilsolitons are classified. Therefore, in higher dimensions, one can easily compute nilsolitons by using the direct sums of lower dimensional nilsolitons. By considering any nilpotent Lie algebra of dimension less than or equal to 6 is an Einstein nil-radical, and that all nilsoliton metric Lie algebras are classified in dimension 7 , we focus on studying indecomposable algebras in this paper.

This paper can be considered as a continuation paper to our last two papers. The first of these papers, we defined an algorithm which prunes algebras with non-simple derivation and with non-singular Gram matrix [19]. But we have not considered the Jacobi identity. The second of these papers, we present some important theorems to construct and solve the Jacobi identity. Also, we presented some other theorems regarding to the other structural elements of a nilsoliton [20]. In this study, we construct an algorithm with the help of the theoretical approach in our last paper. By this study, we have all nilsolitons with nullity $0,1,2$ and 3 in dimension 8 , because we have already classified 8 dimensional nilsoliton metric Lie algebras with simple 
derivation, where the corresponding Gram matrix is nonsingular [15]. In dimension 8, the classifications of such nilsoliton metric Lie algebras can be found in the following theorem:

Theorem 1.1. Let $(\eta,\langle\rangle$,$) be an 8-dimensional nilsoliton metric Lie algebra with simple nilsoliton$ derivation D. If the corresponding canonical Gram matrix $U$ has nullity 1,2 or 3 , then $(\eta,\langle\rangle$,$) is homothetic$ to precisely one of the nilsolitons of dimension 8 that are listed in Tables 1-3.

Proof. The proof of Theorem 1.1 relies on the algorithmic method that is implemented in the computing and programming environment MATLAB.

This paper consists of six sections. In the second section, we present preliminary background to construct the symbolic computation algorithm. In the third section, we present algorithms and we give some examples to illustrate the steps of them. In the fourth section, we present some concluding remarks regarding to the computation results. And in fifth section, we present the notations we have used in classification tables with the several examples, and in the sixth section, we present classification tables.

We use MATLAB R2020b for the implementation of the computational process.

\section{PRELIMINARIES}

Let $(\eta,\langle\rangle$,$) be a metric algebra, with \mu \in \Lambda^{2} \eta \otimes \eta *$, and $\mathrm{B}=\left\{X_{i}: 1 \leq i \leq n\right\}$ be an ordered $\langle$,$\rangle -$ orthonormal basis of $\eta_{\mu}$. The nil-Ricci endomorphism $\operatorname{Ric}_{\mu}$ is defined as $\left\langle\operatorname{Ric}_{\mu} X, Y\right\rangle=\operatorname{ric}_{\mu}(X, Y)$, where $X, Y \in \eta$, and

$\operatorname{ric}_{\mu}(X, Y)=-\frac{1}{2} \sum_{i=1}^{n}\left\langle\left[X, X_{i}\right],\left[Y, X_{i}\right]\right\rangle+\frac{1}{4}\left\langle\left[X_{i}, X_{j}\right], X\right\rangle\left\langle\left[X_{i}, X_{j}\right], Y\right\rangle$.

If $\eta$ is a nilpotent Lie algebra, then the nil-Ricci endomorphism is the Ricci endomorphism. Throughout this paper, we call the orthonormal basis as a Ricci eigenvector basis, if it consists of eigenvectors for the nil-Ricci endomorphism Ric $_{\mu}$.

Let $\eta$ be a nilpotent Lie algebra. $\eta$ and $r$ are called an r-step nilpotent Lie algebra and the nilpotency index of $\eta$ respectively if $\eta^{r}=0$, and $\eta^{r-1} \neq 0$. Here the lower central series for the Lie algebra is defined by $\eta^{0}=\eta$ and $\eta^{k}=\left[\eta, \eta^{k}\right]$ for each for $k \geq 1$.

Let $\Lambda=\left\{(i, j, k): \alpha_{i j}^{k} \neq 0, i<j<k\right\}$ be a finite set that indexes the set of nonzero structure constants corresponding to a Lie algebra, ignoring repetitions due to skew-symmetry. For $1 \leq i, j, k \leq n$, we define $1 \times n$ row vector $y_{i j}^{k}=\epsilon_{i}^{T}+\epsilon_{j}^{T}-\epsilon_{k}^{T}$, where $\left\{\epsilon_{i}\right\}_{1 \leq i \leq n}$ is the standard orthonormal basis for $I R^{n}$. We call the vectors in $\left\{y_{i j}^{k}:(i, j, k) \in \Lambda\right\}$ as root vectors for $\Lambda$. Let $y_{1}, y_{2}, \ldots, y_{r}$ (where $\mathrm{r}=|\Lambda|$ ) be an enumeration of the root vectors in dictionary order. Writing each root vectors as the rows, we create a matrix $\mathrm{Y}$, which we call "root matrix. It can easily be seen that $\mathrm{Y}$ is an $r \times n$ matrix. On the other hand, the Gram matrix $\mathrm{U}$ is the $r \times r$ matrix defined by $U=Y Y^{T}$. It clearly is a symmetric matrix, with each $(\mathrm{i}, \mathrm{j})$ entry it is the inner product of the $i^{t h}$ and $j^{t h}$ row vectors in Y. From Theorem 5 in [21] we know that $\mathrm{U}$ is a matrix where it's all diagonal entries are 3 and its off-diagonal entries are in the set $\{-2,-1,0,1,2\}$.

To classify the Lie algebras, we need to fix our basis to create the possible Lie brackets for nilsolitons. In our paper, we use "nice" basis which was defined by Nikolayevski in [6]. We use this type of basis in our classifications. This way our Gram matrices corresponding to metric nilpotent Lie algebras does not have a 2 or -2 as an entree in their Gram matrices (Lemma 2 in [15]).

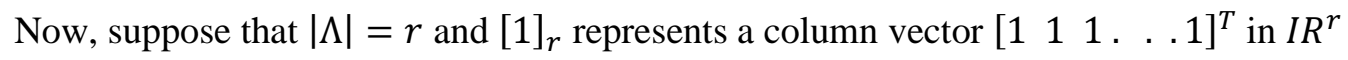


Theorem 2.1. (Theorem 1 in [21]) Let $\eta$ be a nonabelian metric algebra with the basis B which consists of Ricci eigen vectors. Let $\mathrm{U}$ and $\left[\alpha^{2}\right]$ be the Gram matrix and the structure vector for with respect to B. Then $\eta$ satisfies the nilsoliton condition with nilsoliton constant if and only if $U\left[\alpha^{2}\right]=2 \beta[1]_{r}$.

Above theorem indicates a Lie algebra $\eta$ admits a nilsoliton metric if and only if the linear system $\mathrm{U} v=$ $[1]_{r}$ has a solution $\mathrm{v} \in \mathrm{IR}^{\mathrm{r}}$ where all entries are positive real numbers.

\section{ALGORITHM}

In this section, we present three algorithms that we have used in our classifications. Algorithm Index and Algorithm Rank provide algorithm to compute the index and rank of $\eta$ respectively. Algorithm JI provides construction and solution to the Jacobi Identity(s) for corresponding index set, and finally the Main Algorithm provides all necessary steps for the classifications.

\subsection{Algorithm for Computation of the Index and Rank}

We start with the definition of the index of a Lie algebra:

Definition 3.1. Let $X \in \eta, a d_{X}$ denotes the adjoint representation and denotes the dual of the Lie algebra $\eta$. Then the skew symmetric bilinear form $\Psi_{f}$ where $f \in \eta *$ is defined by

$\Psi_{f}: \eta \times \eta \rightarrow I R$

$$
(X, Y) \rightarrow \Psi_{f}(X, Y)=f(X, Y) .
$$

The index of $\eta$ is the integer $\inf \left\{\operatorname{dim}\left(\eta_{f}\right): f \in \eta *\right\}$, where $\eta_{f}=\operatorname{ker}\left(\Psi_{f}\right)$ defined by

$\eta_{f}=\{X \in \eta: f([X, Y])=0, \forall Y \in \eta\}$.

Proposition 3.2. (Proposition 4 in [22]) The index of a n-dimensional Lie algebra is the integer

$\operatorname{index}_{\eta}=n-\operatorname{Rank}_{L I(\eta)}\left(\left[X_{i}, X_{j}\right]\right)_{1 \leq i, j \leq n}$

where $\operatorname{LI}(\eta)$ is the quotient field of symmetric algebra $S(\eta)$.

Remark 3.3. Above proposition tells us that the index of a Lie algebra is the nullity of the matrix $L I_{\eta}$ with $(i, j)^{\text {th }}$ entrée as $\left[X_{i}, X_{j}\right]$.

To create the matrix $L I_{\eta}$, we need following properties:

Proposition 3.4. [20] For an $n$-dimensional nilsoliton represented by an index set $\Lambda$ with a nice basis, the matrix $L I_{\eta}$ is an is $n \times n$ matrix with zero diagonal entries whose rank is even. Also, both of its $\mathrm{n}$-th row and column are zero matrices. If the cardinality of the index set $\Lambda$ is $\mathrm{K}$, then then $L I_{\eta}$ has $2 \mathrm{~K}$ nonzero entries.

Now we define an algorithm "Algorithm Index" regarding to the computation of the index of $\eta$ by symbolic methods using computer systems. The input is the index set $\Lambda$, and $n=\operatorname{dim}(\eta)$, and the output is the index of the Lie algebra. In the algorithm, $L I$ is the matrix in Remark 3.3.

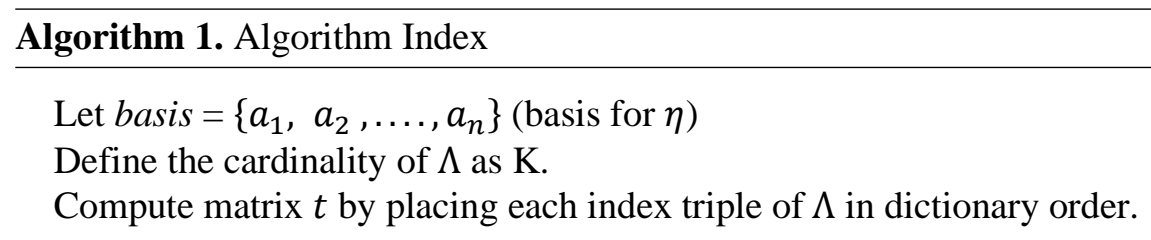




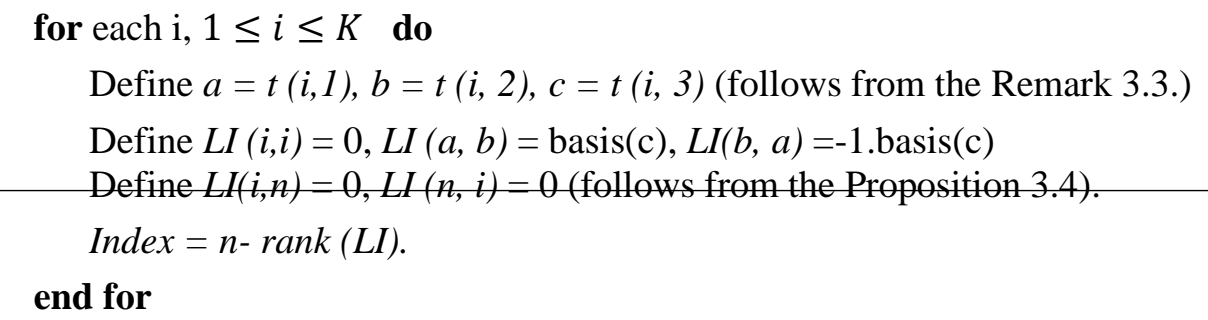

\section{The Rank:}

Let $\eta$ be a metric algebra, and $\operatorname{Der}(\eta)$ be its derivation algebra. The maximal torus is the maximal abelian subalgebra of $\operatorname{Der}(\eta)$ consisting of semi simple elements. The dimension of a maximal torus is called the rank of $\eta$. For the computation of the rank of a nilsoliton, we use following corollary:

Corollary 3.5. (Corollary 3.2.2 in [20]) Let $\eta$ be an n-dimensional nonabelian Lie algebra that admits a simple derivation. Let $\mathrm{B}$ be an eigenvector basis with index set $\Lambda$, and let $\mathrm{Y}$ be the root matrix associated to $\Lambda$. Then the rank of a nilsoliton metric Lie algebra is

$\operatorname{rank}(\eta)=n+\operatorname{nullity}\left(Y Y^{T}\right)-|\Lambda|$

Therefore, to compute the rank of a Lie algebra, we need to compute the nullity of the Gram matrix, $\mathrm{K}=|\Lambda|$, and the dimension of the Lie algebra.

\subsection{Algorithm for Creating and Solving Jacobi Identities}

Now we present theorems which help to create possible Jacobi identities:

Theorem 3.6. [21] Let $\eta$ be an n-dimensional algebra, $B=\left\{X_{i}: 1 \leq i \leq n\right\}$ be a basis for $\eta$. Suppose that a set of nonzero structure constants $\alpha_{i j}^{k}$ relative to B indexed by $\Lambda$, defines a skew symmetric product on $\eta$. Assume that if $(i, j, k) \in \Lambda$, then $\mathrm{i}<\mathrm{j}<\mathrm{k}$. Then the algebra is a Lie algebra if and only

if whenever there exists $\mathrm{m}$ so that the inner product of root vectors $\left\langle y_{i j}^{l}, y_{l k}^{m}\right\rangle=-1$ for triples $(i, j, l),(l, k, m) \in \Lambda$, then the equation

$\sum_{s<m} \alpha_{i j}^{s} \alpha_{s k}^{m}+\alpha_{j k}^{s} \alpha_{s i}^{m}+\alpha_{k i}^{s} \alpha_{s j}^{m}=0$

holds. Moreover, $\left\langle y_{i j}^{l}, y_{l k}^{m}\right\rangle=-1$ if and only if the term $\alpha_{i j}^{l} \alpha_{l k}^{m}$ is nonzero.

To illustrate use of above theorem to create Jacobi identities, we have following example:

Example 3.7. Let $\Lambda=\{(1,2,4),(1,4,5),(1,5,7),(2,3,6),(2,7,8),(3,6,8),(4,5,8)\}$. The nullity of the Gram matrix $U=U_{\Lambda}$ is 1 . Then the solution to $U\left[\alpha^{2}\right]=[1]$ is

$$
\begin{aligned}
& \left(\alpha_{12}^{4}\right)^{2}=x, \quad\left(\alpha_{14}^{5}\right)^{2}=1 / 3,\left(\alpha_{15}^{7}\right)^{2}=4 / 9-x,\left(\alpha_{23}^{6}\right)^{2}=2 / 9, \quad\left(\alpha_{27}^{8}\right)^{2}=1 / 3-x, \\
& \left(\alpha_{36}^{8}\right)^{2}=2 / 9,\left(\alpha_{45}^{8}\right)^{2}=x .
\end{aligned}
$$

Here $x$ stands for the parameter of the solution space. The couple of indexes that leads - 1 entrée as defined in Theorem 3.6 are $(1,2,4)(4,5,8)$ and $(1,5,7)(2,7,8)$. There is a unique Jacobi identity for $\mathrm{m}=$ 8 in the Equation (6) and the Jacobi identity is:

$\alpha_{12}^{4} \alpha_{45}^{8}+\alpha_{15}^{7} \alpha_{72}^{8}=0$ 
Therefore, the Jacobi identity is $\sqrt{x} \sqrt{x}+\sqrt{4 / 9-x} \sqrt{1 / 3-x}=0$, whose solution is $x=4 / 21$, and $\alpha^{2}=$ $\frac{1}{63}(12,21,16,14,9,14,12)$. Since all components of $\alpha^{2}$ is positive, then the given index set corresponds to nilsoliton metric Lie algebra, whose eigenvalues of the nilsoliton derivation is $D=\frac{1}{18}(2,4,5,6,8,9,10,14)$. This nilsoliton metric Lie algebra appears in row 50 of Table 1.

Remark: Considering Theorem 3.6, the Jacobi identity appears only if there exists $\left(i_{1}, j_{1}, k_{1}\right),\left(i_{2}, j_{2}, k_{2}\right) \in$ $\Lambda$ such that $\left\langle y_{i_{1}, j_{1}}^{k_{1}}, y_{i_{2}, j_{2}}^{k_{2}}\right\rangle=-1$. Therefore, if, $\left\langle y_{i_{1}, j_{1}}^{k_{1}}, y_{i_{2}, j_{2}}^{k_{2}}\right\rangle \neq-1$ (which leads to - 1 entrée in the Gram matrix $U$ ) for all couples in the index set, then Jacobi identity is automatically satisfied, i.e., the index set corresponds to a Lie algebra.

Lemma 3.8. (Lemma 2.8 in [15]) Suppose that an n-dimensional nilpotent Lie algebra $\eta$ admits a derivation $\mathrm{D}$ having distinct real positive eigenvalues. Let $\mathrm{B}$ be the eigenvector basis for the derivation $\mathrm{D}$, $\Lambda$ indexes the nonzero structure constants with respect to $\mathrm{B}$, and $\mathrm{Y}$ be the $r \times n$ root matrix for $\Lambda$. If $\mathrm{Y}$ has a maximum rank, then we have

$$
\begin{array}{cl}
\text { i. } & |\Lambda| \leq n-1 \\
\text { ii. } & \text { If }\left(i_{1}, j_{1}, k_{1}\right),\left(i_{2}, j_{2}, k_{2}\right) \in \Lambda \text {, then }\left\langle y_{i_{1}, j_{1}}^{k_{1}}, y_{i_{2}, j_{2}}^{k_{2}}\right\rangle \neq-1 .
\end{array}
$$

Theorem 3.9. (Theorem 3.2 in [19]) Let $\eta$ be an n-dimensional nonabelian nilsoliton with simple derivation where $\mathrm{n}>5$. Suppose that $\mathrm{B}$ is an eigenvector basis regarding to the nilsoliton derivation $D$, and $\Lambda$ indexes the nonzero structure constants with respect to $B$. If the corresponding Gram-matrix $U$ is singular, then $|\Lambda| \geq 5$.

Remark: Considering Lemma 3.8 (ii), if the Gram matrix has a -1 entrée, then the Gram matrix is singular whenever the algebra $\eta$ is a nilsoliton metric Lie algebra with distinct positive eigenvalues. On the other hand, from Lemma 3.8 (i), we conclude that if the cardinality of the index set is greater than $n-1$, then the Gram matrix is singular whenever the algebra $\eta$ is a nilsoliton metric Lie algebra with distinct positive eigenvalues. Also, from Theorem 3.9, the cardinality of the index set being less than 5 corresponds to nonsingular Gram matrices. Since we are considering to classify the nilsoliton metric Lie algebras with distinct positive eigenvalues corresponding to a singular Gram matrix, we will eliminate the index set such that

- The cardinality is greater than 7 corresponding a Gram matrix with no -1 entrée.

- $\quad$ The cardinality is less than 5 .

In order to decide if a given index set corresponds to a Lie algebra, we need to create and solve Jacobi identities for each $\mathrm{m}$ that appears in Equation (6). The following definition gives a procedure to create Jacobi Identities.

Definition 3.10. [20] If there are $r$ product couples in the Jacobi identity for the same $m$ as in Equation (6) such that $(i, j, s),(s, k, m) \in \Lambda$ or $(i, j, s),(s, k, m) \in \Lambda$ then we define

$P_{m}=\left\{p_{1}, p_{2}, \ldots, p_{r}: p_{s}=\left|\alpha_{i j}^{s} \alpha_{s k}^{m}\right|, 1 \leq s \leq r\right\}$

We call $P_{m}$ as the set of product couples for the same \#m in Equation (6). Also, we define the set of all \#m that appears in the Equation (6) as follows:

$A o M=\{m \in I N:(i, j, s),(s, k, m) \in \Lambda$ or $(i, j, s),(s, k, m) \in \Lambda\}$.

Theorem 3.11. [20] Let $\eta$ be an algebra that is indexed by $\Lambda$, and $U=Y Y^{T}$ be the Gram matrix related to $\Lambda$. Let $\mathrm{v}$ belongs to the solution space to the linear system $\mathrm{U} v=[1]$ where $v=\left[\alpha^{2}\right]$ the structure constants with respect to a "nice" basis B (as defined in the introduction section), and $\left|P_{m}\right|=r$. Then the Jacobi identity for each $m \in A o M$ is given by 
$\sqrt{v_{i j}^{s_{1}} v_{s_{1} k}^{m}} \pm \sqrt{v_{j k}^{s_{2}} v_{s_{2} i}^{m}} \pm \sqrt{v_{k i}^{s_{r}} v_{s_{r} j}^{m}}=0$

In the following, we use above definitions and Theorem 3.11 for defining an algorithm that creates Jacobi identity for each $m \in A o M$.

Remark 3.12. The above theorem helps one to create Jacobi identity for each $m \in$ AoM. So far, we create Jacobi Identities in Equation (6) separately for each distinct $\mathrm{m}$ in AoM. In this process one needs to make sure that for all the products $v_{i j}^{s_{1}} v_{s_{1} k}^{m}$ in Equation (9) must have the same $\mathrm{i}, \mathrm{j}$, and $\mathrm{k}$ indices. There can be more than one possible $\{\mathrm{i}, \mathrm{j}, \mathrm{k}\}$ entries in the above equation. In that case, the equation should be written and solved separately. Therefore, besides writing index couples with the same \#m in AoM, we should also divide index couples with the same \#m in terms of their $\{\mathrm{i}, \mathrm{j}, \mathrm{k}\}$ entrees to create all Jacobi Identities to be solved. The following example illustrates this issue.

Example 3.13. Suppose that the index set is $\Lambda=\{(1,2,4),(1,4,5),(1,5,6),(2,6,7),(4,5,7),(3,7,8)\}$. If we compute the \#m's that lead -1 entries in the Gram matrix, $A o M=\{7,8\}$. The couples of index triples that lead $m=8$ are $\{(2,6,7),(3,7,8)\}$ and $\{(4,5,7),(3,7,8)\}$. The first and the second couple of index triples lead to the products $\alpha_{26}^{7} \alpha_{73}^{8}$ and $\alpha_{45}^{7} \alpha_{73}^{8}$ respectively. Therefore, the first index couple represent the Jacobi identity for $\{\mathrm{i}, \mathrm{j}, \mathrm{k}\}=\{2,6,3\}$, and the second index couple represent the Jacobi identity for $\{\mathrm{i}, \mathrm{j}, \mathrm{k}\}=\{4,5,3\}$. They clearly are different, so they both have to be solved separately as $\alpha_{26}^{7} \alpha_{73}^{8}=0$ and $\alpha_{45}^{7} \alpha_{73}^{8}=0$. Clearly at least one of the structure constants is zero, therefore this case should be eliminated.

\section{The Procedure to Create Jacobi Identities}

If there are $\mathrm{r}$ product couples in the Jacobi identity for same $m \in A o M$, to encode those $2^{r-1}-1$ number of sign choices, we define a $\left(2^{r-1}-1\right) \times(r-1)$ matrix denoted by $S C=\left[s_{j S}\right]$ such that for each $j \in\left\{1,2, \ldots, 2^{r-1}-1\right\}$, we have

$s_{j s}= \begin{cases}1 & p_{s}>0 \\ 0 & p_{s}<0\end{cases}$

where $p_{s}= \pm \sqrt{v_{i j}^{s} v_{s k}^{m}}$. The $j^{t h}$ Jacobi identity is calculated by $p_{1}+\sum_{i=1}^{r-1}(-1)^{s_{j i}} p_{i}=0$. For example, if there are 3 product couples for $m \in A o M$, then possible Jacobi identities are

$p_{1}+(-1)^{s_{11}} p_{2}+(-1)^{s_{12}} p_{3}=0$

$p_{1}+(-1)^{s_{21}} p_{2}+(-1)^{s_{22}} p_{3}=0$

$p_{1}+(-1)^{s_{31}} p_{2}+(-1)^{s_{32}} p_{3}=0$

In order $\eta$ to be a Lie algebra, at least one of the above equations has to be satisfied for each $m \in A o M$. If there is more than one entry in $A o M$, then we need to find a common solution of Jacobi identities for all $\mathrm{m}$.

Now we present the algorithm for creating and solving the Jacobi identity(s) for a given an index set $\Lambda$, where its cardinality is $\mathrm{K}$. The input of the algorithm is the index set $\Lambda$, and the outputs are $e q n_{r}$ for all $r \in\{2,3\}$. In the Algorithm JI, eqn $r$ represents the Jacobi identity consisting of $r$ product couples for each $r \in\{2,3\}$, W represents the 0/1 matrix, where each row represents possible characteristic vectors for canonical index sets for nilpotent Lie algebras of dimension $n$ with singular Gram matrix. We note that $r$ cannot be 1 because of the fact that $r=1$ corresponds to a unique product couple in the equation, which leads the fact that at least one of the structure constants is zero. 


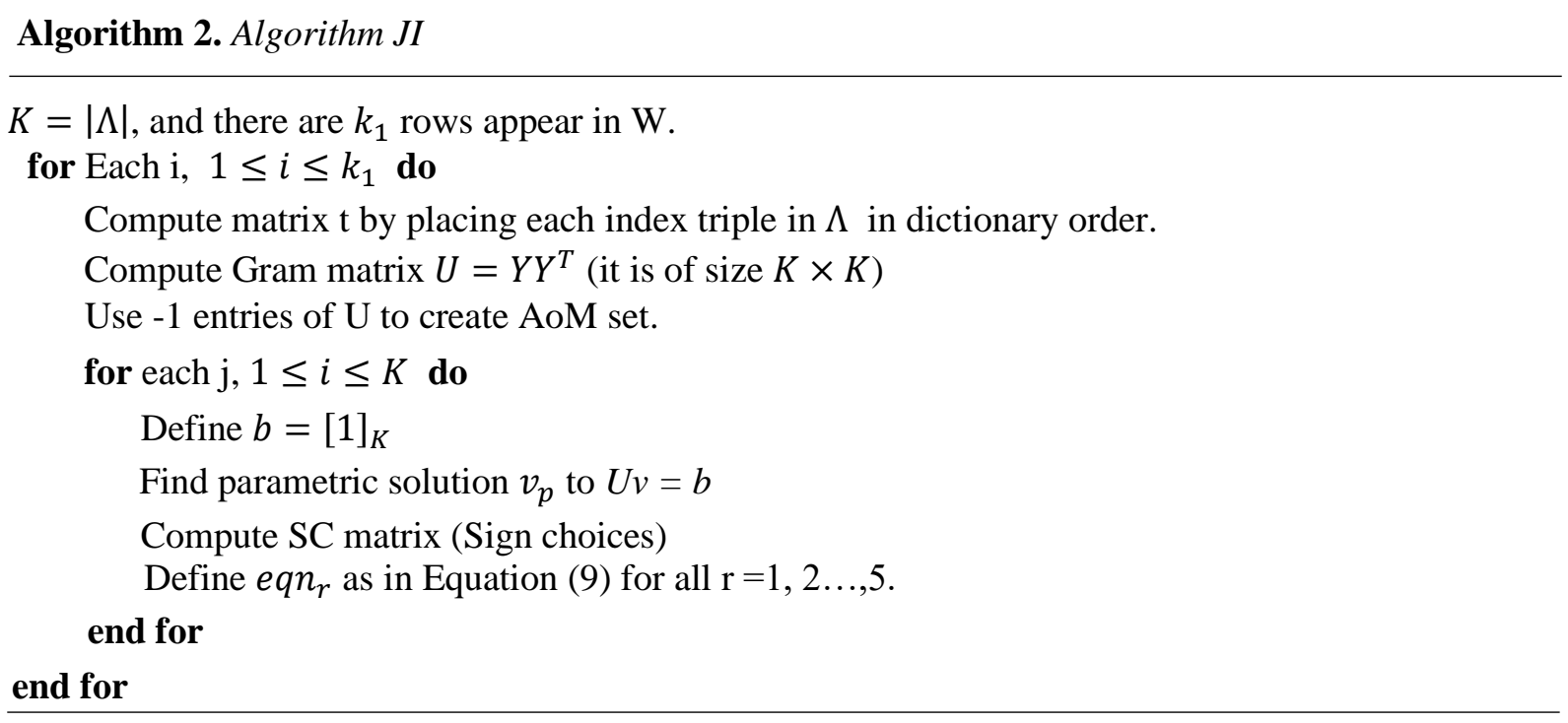

\subsection{The Main Algorithm}

In our previous paper [19], we construct a $0 / 1$ matrix representing all possible characteristic vectors for canonical index sets for an n-dimensional nilpotent Lie algebra with singular Gram matrix. Using theoretical background, we have pruned the rows corresponding to a unique -1 inner product of root vectors, nonsingular Gram matrices, abelian algebras, and non-simple derivations. We also have pruned some of the rows of $\mathrm{W}$ that correspond to algebras that does not satisfy Jacobi identity in the 14th step of the algorithm. For each algebra in the row of W matrix, we first obtain the array of number m's in Equation (6) in the Theorem 3.6. We call the array as Arrayofms which consists of the elements of AoM set. Therefore, different number m's correspond to different equations. Thus, if there is a unique $\mathrm{m}$ for $\Lambda$, there exists a unique product couple $p_{s}=\alpha_{i j}^{S} \alpha_{s k}^{m}$ in $P_{m}$. So, the Jacobi identity turns into $\alpha_{i j}^{S} \alpha_{s k}^{m}=0$, which implies that $\alpha_{i j}^{s}=0$ or $\alpha_{s k}^{m}=0$. This is a contradiction to the assumption that the set $\Lambda$ indexes the nonzero structure constants. Therefore, we eliminate these cases in our algorithm. The following is an example of such case:

Example 3.14. Let $\Lambda=\{(1,2,3),(1,6,7),(3,4,7),(3,5,6),(3,6,8)\}$, then -1 entries are coming from $\{(1,2,3),(3,4,7)\},\{(1,2,3),(3,5,6)\},\{(1,2,3),(3,6,8)\}$ and $\{(1,6,7),(3,5,6)\}$. Then, $A o M=\{6,7,8\}$ respectively. As can be seen, $m=8$ is coming only from indexes $\{(1,2,3),(3,6,8)\}$. So, corresponding Jacobi identity is $\alpha_{12}^{3} \alpha_{36}^{8}=0$. This kind of cases will be eliminated in our procedure.

So far, we have eliminated the rows that correspond to zero structure constants. But this does not eliminate the rows not corresponding to a nilsoliton metric Lie algebra. What we need to do is to compute Jacobi identity equation/equations (which are non-linear equations) for each algebra, and find out if there exists a common solution to the system of non-linear equations.

For this, we first start with dividing the $\mathrm{W}$ matrix in terms of its nullity type. Since we classify nilsolitons with the nullity of the Gram matrix is $1-3$, we need to create three submatrices of W, WNullity1 to WNullity 3 which consist of the rows of $\mathrm{W}$. Please note that the maximum nullity is 5 , therefore one needs to create WNullity4 and WNullity5 to classify nilsolitons with the corresponding Gram matrices have nullity 4 or 5 .

Now we present the algorithm that we have used for our classifications. The input is the Prunned W matrix in [19], and the outputs are the matrices $W_{s}$ and $\mathrm{V}$ where each row of these matrices corresponds to a nilsoliton with simple nilsoliton derivation, and the common solution to the Jacobi identities respectively. Each row of $\mathrm{V}$ provides the squares of structure constants to the corresponding nilsoliton. 
The main algorithm of this study is the following one:

Input: Prunned W ([19])

Output: $\mathrm{W}_{\mathrm{s}}$ and $\mathrm{V}_{\mathrm{s}}$

\section{Algorithm 3. Find Matrix $\mathrm{W}_{\mathrm{s}}$ and $\mathrm{V}_{\mathrm{s}}$}

1. Use Theorem 3.9 to eliminate the rows of $W$ that corresponds to an index set where $|\Lambda|<5$

2. For each submatrix, compute AoM, and sub-index set $\Lambda_{\mathrm{s}}$

3. Check if each couple of index triples belongs to the same Jacobi Identity (Remark 3.12)

4. Use Algorithm JI to create system of Jacobi Identities for each entry in AoM

5. Find common solution $v$ of Jacobi Identities for each entree in AoM and find square of the structure constants in $\mathrm{v}$.

6. if $v \leq 0$ then

Delete the row from $\mathrm{W}$

else

Save the solution in Vs matrix

end if

7. $\mathrm{W}_{\mathrm{s}}=\mathrm{W}$

Remark 3.15. To find the solution of the system of Jacobi identities, we use solve and vpasolve commands for exact and VPA solutions respectively in MATLAB.

Remark 3.16. After finding $\mathrm{W}_{\mathrm{s}}$, we only need to return all structural elements of the nilsolitons.

\section{CONCLUSION}

Remark 4.1. After applying above algorithm, we find a counterexample of the reverse of Lemma 3.8. Therefore, if none of the inner product of root vectors is -1 does not imply that the Gram matrix of the nilsoliton is nonsingular. Additionally, if the cardinality of the index set $|\Lambda| \leq n-1$ does not imply that the Gram matrix is nonsingular. The following is one of the examples of this case.

Example 4.2. Suppose that $\eta$ be an algebra which is indexed by the following index set:

$\Lambda=\{(1,2,5),(1,3,6),(1,5,7),(1,6,8),(2,4,7),(2,5,8),(3,4,8)\}$.

The corresponding Gram matrix is a singular with nullity $=1$. Also, it does not have any -1 entry. From Theorem 3.6, it is a Lie algebra. Also, since the solution space of $\mathrm{Uv}=[1]$ is

$$
\{v=(x+1 / 19,6 / 19-x, x, 5 / 19-x, 5 / 19-x, 3 / 19, x): 0<x<5 / 19\}
$$

from [20] that it is a nilsoliton metric Lie algebra with the magnitudes of the structure constants

$$
\begin{aligned}
& \left|\alpha_{12}^{5}\right|=\sqrt{x+1 / 19}, \quad\left|\alpha_{13}^{6}\right|=\sqrt{6 / 19-x}, \quad\left|\alpha_{15}^{7}\right|=\sqrt{x},\left|\alpha_{16}^{8}\right|=\sqrt{5 / 19-x}, \\
& \left|\alpha_{24}^{7}\right|=\sqrt{5 / 19-x}, \quad\left|\alpha_{25}^{8}\right|=\sqrt{3 / 19},\left|\alpha_{34}^{8}\right|=\sqrt{x} .
\end{aligned}
$$

From Theorem 2.5 in [15], the eigenvalues of the derivation are the same for all choices of the parameter $\mathrm{x}$. Therefore, without finding exact solution, we only need to use one of the solutions to $\mathrm{Uv}=[1]$ which is 


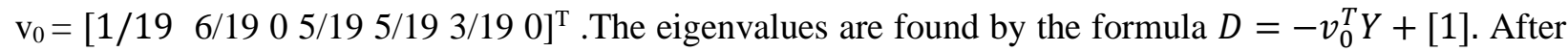
the computation, we have

$\mathrm{D}=[7 / 19$ 10/19 13/19 14/19 17/19 20/19 24/19 27/19].

Therefore, the given index set corresponds to a nilsoliton metric Lie algebra with distinct positive eigenvalues of the nilsoliton derivation of type $7<10<13<14<17<20<24<27$. Thus, the index set of this nilsoliton metric Lie algebra with simple derivation corresponds to a singular Gram matrix $U$ which does not have any -1 entrée and $|\Lambda|=n-1=7$.

Remark 4.3. After running the algorithm, we could not find any 2-step nilpotent Lie algebra with soliton metric in dimension 8 where the nullity of the corresponding Gram matrices is in $\{0,1,2,3\}$. As we combine this result with the results of our previous paper [15], we conclude that there is no 2-step nilpotent Lie algebra endowed with a soliton metric in dimension 8, where the nullity of its Gram matrix is $0,1,2$ or 3 .

\section{CLASSIFICATIONS}

Classification results for dimension 8 appear at the end of this section. By this classification, we complete all nilsoliton metric Lie algebras with the nullity of their Gram matrices are in $\{0,1,2,3\}$, and with simple nilsoliton derivation in dimension 8. Please note that the Lie algebras appear in Tables 1, 2 and 3 are the ones whose nullity of the Gram matrix is 1, 2 and 3 respectively. We use vector notation to represent Lie algebra structures, as we did in $[15,17]$.

\section{Notation:}

1. Lie Bracket column illustrates both structure constants and how Lie bracket functions on a given ordered eigenvector bases $\left\{X_{i}\right\}_{i=1}^{8}$. In the following, we give an example to illustrate how to read the tables in this section:

Example 5.1. The Lie bracket type:

$$
(0,0,2 \sqrt{3} .12,0,2 \sqrt{5} .13, \sqrt{15} .15,2 \sqrt{3} .24,2 \sqrt{3} .26+\sqrt{15} .35)
$$

illustrates the structure constants together with Lie brackets as follows:

$$
\begin{aligned}
& {\left[X_{1}, X_{2}\right]=2 \sqrt{3} X_{3},\left[X_{1}, X_{3}\right]=2 \sqrt{5} X_{5},\left[X_{1}, X_{5}\right]=\sqrt{15} X_{6}} \\
& {\left[X_{2}, X_{4}\right]=2 \sqrt{3} X_{7},\left[X_{2}, X_{6}\right]=2 \sqrt{3} X_{8},\left[X_{3}, X_{5}\right]=\sqrt{15} X_{8}}
\end{aligned}
$$

The eigenvalues of the nilsoliton derivation are $\frac{1}{120}(13,24,37,48,50,63,72,87)$. Therefore, the nilsoliton is of type $13<24<37<48<50<63<72<87$.

2. $|\mathrm{AoM}|$ shows how many different \#m in Equation (6) appears in the Lie algebra. For example, for the Lie bracket type

$$
(0,0,0, \sqrt{10} .13, \sqrt{11} .14, \sqrt{5} .15+\sqrt{10} .23, \sqrt{11} .16+\sqrt{11} .24, \sqrt{10} .17+\sqrt{10} .25)
$$

$|\mathrm{AoM}|$ is 2, because there are two different $m \in A o M$ which are [7 8]. That means, the number m's in the Equation (6) are 7 and 8. Therefore there are two Jacobi identities as the following:

For $\mathrm{m}=7$, the Jacobi identity is $\alpha_{23}^{6} \alpha_{16}^{7}-\alpha_{13}^{4} \alpha_{24}^{7}=0$, where $\left|\alpha_{23}^{6}\right|=\sqrt{10},\left|\alpha_{16}^{7}\right|=\sqrt{11}$, $\left|\alpha_{13}^{4}\right|=\sqrt{10}$, and $\left|\alpha_{24}^{7}\right|=\sqrt{11}$.

For $\mathrm{m}=8$, the Jacobi identity is $\alpha_{24}^{7} \alpha_{17}^{8}-\alpha_{14}^{5} \alpha_{25}^{8}=0$, where $\left|\alpha_{24}^{7}\right|=\sqrt{11}$, 
$\left|\alpha_{17}^{8}\right|=\sqrt{10} \quad\left|\alpha_{14}^{5}\right|=\sqrt{11} \quad\left|\alpha_{25}^{8}\right|=\sqrt{10}$

in which both are satisfied. Therefore, since both Jacobi identities are satisfied, it is a Lie algebra. On the other hand, this solution leads to the squares of the structure constants vector

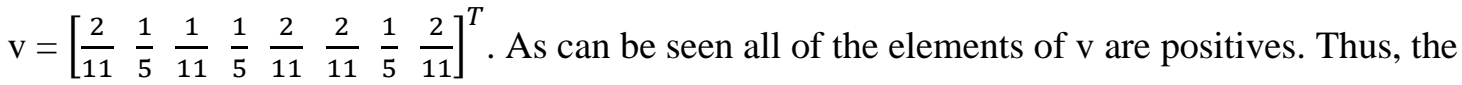
Lie algebra is nilsoliton.

3. The E/VPA column, the letter E shows that the solution of the Jacobi identity is exact. VPA shows that the solution is approximated using VPA (Variable-precision arithmetic). Please note that VPA evaluates each element of the symbolic input $\mathrm{x}$ to at least 32 significant digits. There is also symbol appears in \#68 and \#69 in Table 1, meaning that there is no -1 entry in their Gram matrix, therefore the corresponding Jacobi identity is always satisfied for any real number such that all entries of the solution are positive numbers.

4. The Rank column illustrates the dimension of maximal torus of derivations.

5. The Index column illustrates the index of the Lie algebra.

6. The NI column illustrates the nilpotency index of the Lie algebra, i.e. the length of the lower central series for the Lie algebra.

Table 1. 8-dimensional nilsoliton metric Lie algebras-nullity 1

\begin{tabular}{|c|c|c|c|c|c|c|c|}
\hline & Lie Bracket & Derivation Type & |AoM & $\begin{array}{l}\text { E/ } \\
\text { VPA }\end{array}$ & Rank & Index & NI \\
\hline 1 & $\begin{array}{l}(0,0,0,0,6 \cdot 13, \sqrt{30} \cdot 23, \sqrt{22} \cdot 15+ \\
\sqrt{22} \cdot 24, \sqrt{30} \cdot 16+5.25)\end{array}$ & $\begin{array}{l}5<6<7<11<12< \\
13<17<18\end{array}$ & 1 & $\mathrm{E}$ & 3 & 4 & 3 \\
\hline 2 & $\begin{array}{l}(0,0,0,0, \sqrt{2} \cdot 13, \sqrt{2} \cdot 15+ \\
1.23, \sqrt{2} \cdot 24, \sqrt{2} \cdot 16+1.25)\end{array}$ & $\begin{array}{l}2<4<5<6< \\
7<9<10<11\end{array}$ & 1 & $\mathrm{E}$ & 3 & 4 & 4 \\
\hline 3 & $\begin{array}{c}(0,0,0,0,2 \cdot 13, \sqrt{5} .23,2 \cdot 14,2 \cdot 16 \\
+\sqrt{5} .25)\end{array}$ & $\begin{array}{l}8<10<11<16<19< \\
21<24<29\end{array}$ & 1 & $\mathrm{E}$ & 4 & 4 & 3 \\
\hline 4 & $\begin{array}{l}(0,0,0,0,3 \cdot 13, \sqrt{14} \cdot 14, \sqrt{14} \cdot 16+ \\
2 \sqrt{3} \cdot 23,2 \sqrt{3} \cdot 17+4.25)\end{array}$ & $\begin{array}{l}2<5<6<7<8<9< \\
11<13\end{array}$ & 1 & $\mathrm{E}$ & 3 & 4 & 4 \\
\hline 5 & $\begin{array}{l}(0,0,0,0,3 \sqrt{2} \cdot 13, \sqrt{11} \cdot 14+ \\
\sqrt{22} \cdot 23,2 \sqrt{7} \cdot 15+ \\
\sqrt{14} \cdot 24, \sqrt{22} \cdot 17+2 \sqrt{7} \cdot 26)\end{array}$ & $\begin{array}{l}10<15<23<28<33< \\
38<43<53\end{array}$ & 1 & $\mathrm{E}$ & 2 & 4 & 4 \\
\hline 6 & $\begin{array}{l}(0,0,0,0,3 \sqrt{3} \cdot 13, \sqrt{14} \cdot 14+ \\
\sqrt{23} \cdot 23, \sqrt{14} .15+4.24,3 \sqrt{3} .16+ \\
\sqrt{23} .25)\end{array}$ & $\begin{array}{l}10<15<18<23< \\
28<33<38<43\end{array}$ & 1 & $\mathrm{E}$ & 2 & 4 & 3 \\
\hline 7 & $\begin{array}{l}(0,0,0,6 \sqrt{5} \cdot 13, \sqrt{165} \cdot 23 \\
\sqrt{161} \cdot 14,4 \sqrt{6} .15+ \\
2 \sqrt{22} \cdot 24, \sqrt{161} \cdot 25+\sqrt{46} .34)\end{array}$ & $\begin{array}{l}12<13<14<26<27< \\
38<39<40\end{array}$ & 1 & $\mathrm{E}$ & 2 & 4 & 3 \\
\hline 8 & $\begin{array}{l}(0,0,0, \sqrt{22} \cdot 13,2 \sqrt{7} \cdot 23,2 \sqrt{7} \cdot 14, \\
\sqrt{11} \cdot 15+\sqrt{14} \cdot 24,3 \sqrt{2} \cdot 16+ \\
\sqrt{22} \cdot 25)\end{array}$ & $\begin{array}{l}30<45<59<89< \\
104<119<134<149\end{array}$ & 1 & $\mathrm{E}$ & 2 & 4 & 4 \\
\hline 9 & $\begin{array}{l}(0,0,0, \sqrt{21} \cdot 13, \sqrt{22} \cdot 14,4 \sqrt{2} \cdot 15 \\
\sqrt{22} .16+\sqrt{29} \cdot 23, \sqrt{21} \cdot 17+ \\
\sqrt{29} .24)\end{array}$ & $\begin{array}{l}5<20<22<27< \\
32<37<42<47\end{array}$ & 1 & $\mathrm{E}$ & 2 & 4 & 6 \\
\hline
\end{tabular}




\begin{tabular}{|c|c|c|c|c|c|c|c|}
\hline $\mathbf{1 0}$ & $\begin{array}{l}(0,0,0,3 \cdot 12,0,3 \cdot 23,2 \sqrt{2} \cdot 14, \sqrt{7} \cdot 16 \\
+\sqrt{2} \cdot 25+\sqrt{7} \cdot 34)\end{array}$ & $\begin{array}{l}7<9<11<16<18< \\
20<23<27\end{array}$ & 1 & $\mathrm{E}$ & 3 & 2 & 3 \\
\hline 11 & $\begin{array}{l}(0,0,0, \sqrt{3} .12,0,2.14,2.16+ \\
\sqrt{3} .23, \sqrt{3} .17+\sqrt{2} .25+\sqrt{3} .34)\end{array}$ & $\begin{array}{l}2<5<6<7<8<9< \\
11<13\end{array}$ & 1 & $\mathrm{E}$ & 2 & 2 & 5 \\
\hline 12 & $\begin{array}{l}(0,0,0, \sqrt{5} .12,0, \sqrt{3} .14+ \\
2.23, \sqrt{2} \cdot 15+\sqrt{3} \cdot 24, \sqrt{5} .16+ \\
2.34)\end{array}$ & $\begin{array}{l}7<10<14<17< \\
20<24<27<31\end{array}$ & 1 & $\mathrm{E}$ & 2 & 4 & 4 \\
\hline 13 & $\begin{array}{l}(0,0,0,6 \sqrt{10} \cdot 12,4 \sqrt{39} \cdot 23 \\
\sqrt{574} \cdot 14, \sqrt{255} \cdot 15+ \\
\sqrt{442} \cdot 34, \sqrt{451} \cdot 16+\sqrt{451} \cdot 25)\end{array}$ & $\begin{array}{l}19<24<33<43< \\
57<62<76<81\end{array}$ & 1 & $\mathrm{E}$ & 2 & 2 & 4 \\
\hline 14 & $\begin{array}{l}(0,0,0, \sqrt{819} \cdot 12,6 \sqrt{23} .14 \\
\sqrt{975} \cdot 23, \sqrt{805} \cdot 15+ \\
\sqrt{253} \cdot 24, \sqrt{630} .16+\sqrt{750} .34)\end{array}$ & $\begin{array}{l}45<90<104<135< \\
180<194<225< \\
239\end{array}$ & 1 & $\mathrm{E}$ & 2 & 4 & 4 \\
\hline 15 & $\begin{array}{l}(0,0,0,17 \sqrt{6} \cdot 12, \sqrt{1353} \cdot 14 \\
7 \sqrt{41} \cdot 15+6 \sqrt{39} \cdot 23 \\
2 \sqrt{410} \cdot 24, \sqrt{1978} \cdot 16+6 \sqrt{43} \cdot 34)\end{array}$ & $\begin{array}{l}51<107<153<158< \\
209<260<265< \\
311\end{array}$ & 1 & $\mathrm{E}$ & 2 & 4 & 5 \\
\hline 16 & $\begin{array}{l}(0,0,0, \sqrt{6} .12,3.13, \sqrt{10} .15 \\
\sqrt{10} .24, \sqrt{5} .16+2.25+\sqrt{6} .34)\end{array}$ & $\begin{array}{l}2<4<5<6<7<9< \\
10<11\end{array}$ & 1 & $\mathrm{E}$ & 2 & 2 & 4 \\
\hline 17 & $\begin{array}{l}(0,0,0, \sqrt{6} .12,5.13, \sqrt{10} .15+ \\
2.23, \sqrt{10} .24,3.16+\sqrt{6} .34) \\
\end{array}$ & $\begin{array}{l}2<4<5<6<7<9< \\
10<11\end{array}$ & 1 & $\mathrm{E}$ & 2 & 4 & 4 \\
\hline 18 & $\begin{array}{l}(0,0,0, \sqrt{21} \cdot 12, \sqrt{29} \cdot 13,4 \sqrt{2} \cdot 15 \\
\sqrt{22} \cdot 16+\sqrt{22} \cdot 24, \sqrt{21} \cdot 25+ \\
\sqrt{29} \cdot 34)\end{array}$ & $\begin{array}{l}17<37<40<54< \\
57<74<91<94\end{array}$ & 1 & $\mathrm{E}$ & 2 & 2 & 4 \\
\hline 19 & $\begin{array}{l}(0,0,0,3 \cdot 12, \sqrt{7} \cdot 13, \sqrt{2} \cdot 23 \\
4 \sqrt{2} \cdot 14,3 \cdot 25+\sqrt{7} \cdot 34)\end{array}$ & $\begin{array}{l}7<9<11<16<18< \\
20<23<27\end{array}$ & 1 & $\mathrm{E}$ & 3 & 4 & 3 \\
\hline 20 & $\begin{array}{l}(0,0,0,3 \cdot 12,2 \sqrt{5} \cdot 13,3 \sqrt{2} .14+ \\
4.23,3 \sqrt{2} \cdot 24,5.16+2 \sqrt{5} .25)\end{array}$ & $\begin{array}{l}4<5<8<9<12< \\
13<14<17\end{array}$ & 1 & $\mathrm{E}$ & 2 & 4 & 4 \\
\hline 21 & $\begin{array}{l}(0,0,0,2 \sqrt{3} \cdot 12,3 \cdot 13, \sqrt{14} \cdot 14 \\
\sqrt{14} \cdot 16,4 \cdot 25+2 \sqrt{3} \cdot 34)\end{array}$ & $\begin{array}{l}2<5<6<7<8<9< \\
11<13\end{array}$ & 1 & $\mathrm{E}$ & 3 & 2 & 4 \\
\hline 22 & $\begin{array}{l}(0,0,0,3 \sqrt{91} \cdot 12, \sqrt{630} \cdot 13 \\
6 \sqrt{23} \cdot 14, \sqrt{805} \cdot 16+ \\
\sqrt{253} \cdot 24,5 \sqrt{39} \cdot 25+5 \sqrt{30} \cdot 34)\end{array}$ & $\begin{array}{l}45<90<119<135< \\
164<180<225< \\
254\end{array}$ & 1 & $\mathrm{E}$ & 2 & 2 & 4 \\
\hline 23 & $\begin{array}{l}(0,0,0,3 \sqrt{3} \cdot 12,3 \sqrt{3} \cdot 13, \sqrt{14} \cdot 14 \\
\sqrt{14} \cdot 15+4 \cdot 24, \sqrt{23} \cdot 25+ \\
\sqrt{23} \cdot 34)\end{array}$ & $\begin{array}{l}5<7<9<12<14< \\
17<19<21\end{array}$ & 1 & $\mathrm{E}$ & 2 & 4 & 3 \\
\hline 24 & $\begin{array}{l}(0,0,0,2 \sqrt{42} \cdot 12,9 \sqrt{2} \cdot 13,3 \sqrt{11} \cdot 14 \\
\sqrt{77} \cdot 15+\sqrt{110} \cdot 24, \sqrt{33} .16+ \\
\sqrt{140} \cdot 25+3 \sqrt{5} .34)\end{array}$ & $\begin{array}{l}2<3<4<5<6<7< \\
8<9\end{array}$ & 1 & $\mathrm{E}$ & 1 & 2 & 4 \\
\hline 25 & $\begin{array}{l}(0,0,0, \sqrt{31} \cdot 12,2 \cdot 13,3 \sqrt{2} .14+ \\
5.23, \sqrt{14} .15+2 \sqrt{5} .24 \\
\sqrt{31} .16+5.34)\end{array}$ & $\begin{array}{l}2<3<4<5<6<7< \\
8<9\end{array}$ & 1 & $\mathrm{E}$ & 1 & 4 & 4 \\
\hline 26 & $\begin{array}{l}(0,0,0, \sqrt{33} \cdot 12,9 \sqrt{2} \cdot 13,3 \sqrt{11} \cdot 14+ \\
3 \sqrt{15} \cdot 23, \sqrt{77} \cdot 15+ \\
\sqrt{110} \cdot 24,2 \sqrt{42} \cdot 16+\sqrt{140} \cdot 25)\end{array}$ & $\begin{array}{l}2<3<4<5<6<7< \\
8<9\end{array}$ & 1 & $\mathrm{E}$ & 1 & 4 & 4 \\
\hline 27 & $\begin{array}{l}(0,0, \sqrt{11685} \cdot 12,0,2 \sqrt{3399} \cdot 13 \\
4 \sqrt{1030} \cdot 23,14 \sqrt{103} \cdot 15+\end{array}$ & $\begin{array}{l}2<3<5<6<7<8< \\
9<11\end{array}$ & 1 & $\mathrm{E}$ & 1 & 2 & 5 \\
\hline
\end{tabular}




\begin{tabular}{|c|c|c|c|c|c|c|c|}
\hline & $\begin{array}{l}\sqrt{7885} .24, \sqrt{13653} .17+ \\
\sqrt{12566} .26+\sqrt{9215} .34)\end{array}$ & & & & & & \\
\hline 28 & $\begin{array}{l}(0,0, \sqrt{287} \cdot 12,0, \sqrt{176} .13 \\
\sqrt{456} \cdot 23, \sqrt{287} \cdot 15+ \\
\sqrt{246} \cdot 24, \sqrt{374} \cdot 16+\sqrt{323} \cdot 25)\end{array}$ & $\begin{array}{l}15<19<34<45< \\
49<53<64<68\end{array}$ & 1 & $\mathrm{E}$ & 2 & 4 & 4 \\
\hline 29 & $\begin{array}{l}(0,0, \sqrt{46} \cdot 12,0,6 \sqrt{5} \cdot 13, \sqrt{161} \cdot 15 \\
+4 \sqrt{6} .23, \sqrt{161} \cdot 24, \sqrt{165} .16 \\
+2 \sqrt{22} .25)\end{array}$ & $\begin{array}{l}7<14<21<24<28< \\
35<38<42\end{array}$ & 1 & $\mathrm{E}$ & 2 & 4 & 5 \\
\hline 30 & $\begin{array}{l}(0,0, \sqrt{22} \cdot 12,0, \sqrt{30} \cdot 13,6.23 \\
\sqrt{22} \cdot 14,5 \cdot 16+\sqrt{30} .25)\end{array}$ & $\begin{array}{l}4<5<9<11<13< \\
14<15<18\end{array}$ & 1 & $\mathrm{E}$ & 3 & 4 & 4 \\
\hline 31 & $\begin{array}{l}(0,0, \sqrt{14} \cdot 12,0,3 \sqrt{3} \cdot 13, \sqrt{14} \cdot 14+ \\
\sqrt{23} \cdot 23,4.24,3 \sqrt{3} \cdot 16+\sqrt{23} \cdot 25)\end{array}$ & $\begin{array}{l}20<23<43<46<63< \\
66<69<86\end{array}$ & 1 & $\mathrm{E}$ & 2 & 4 & 4 \\
\hline 32 & $\begin{array}{l}(0,0, \sqrt{253} \cdot 12,0,3 \sqrt{70} \cdot 13 \\
6 \sqrt{23} \cdot 14, \sqrt{105} \cdot 16+ \\
\sqrt{819} \cdot 23,5 \sqrt{30} \cdot 17+\sqrt{975} \cdot 25)\end{array}$ & $\begin{array}{ll}37<90< & 127< \\
143<164< & 180< \\
217<254 & \end{array}$ & 1 & $\mathrm{E}$ & 2 & 4 & 4 \\
\hline 33 & $\begin{array}{l}(0,0, \sqrt{442} \cdot 12,0, \sqrt{451} \cdot 13 \\
\sqrt{255} \cdot 14+ \\
\sqrt{451} \cdot 23, \sqrt{574} \cdot 15, \sqrt{624} \cdot 26+ \\
6 \sqrt{10} \cdot 34)\end{array}$ & $\begin{array}{l}17<22<39<44< \\
56<61<73<83\end{array}$ & 1 & $\mathrm{E}$ & 2 & 2 & 4 \\
\hline 34 & $\begin{array}{l}(0,0,4 \cdot 12,0,3 \sqrt{3} \cdot 13, \sqrt{14} .14 \\
\sqrt{14} .15+3 \sqrt{3} .23, \sqrt{23} \cdot 17+ \\
\sqrt{23} \cdot 25)\end{array}$ & $\begin{array}{l}7<14<21<27<28< \\
34<35<42\end{array}$ & 1 & $\mathrm{E}$ & 2 & 4 & 5 \\
\hline 35 & $\begin{array}{l}(0,0, \sqrt{14820} \cdot 12,0, \sqrt{15774} .13 \\
\sqrt{7885} .14+ \\
\sqrt{19120} .23, \sqrt{23422} .15 \\
\sqrt{6692} .17+\sqrt{22464} .26+ \\
\sqrt{11952} .34)\end{array}$ & $\begin{array}{l}2<3<5<6<7<8< \\
9<11\end{array}$ & 1 & $\mathrm{E}$ & 1 & 2 & 5 \\
\hline 36 & $\begin{array}{l}\quad(0,0,2 \sqrt{255} \cdot 12,0, \sqrt{5610} \cdot 13 \\
\sqrt{3071} \cdot 14+20 \sqrt{17} \cdot 23 \\
\sqrt{8330} .15+4 \sqrt{249} .24 \\
2 \sqrt{1591} \cdot 17+8 \sqrt{129} .26)\end{array}$ & $\begin{array}{l}2<3<5<6<7<8< \\
9<11\end{array}$ & 1 & $\mathrm{E}$ & 1 & 4 & 5 \\
\hline 37 & $\begin{array}{l}(0,0, \sqrt{1533} \cdot 12,0, \sqrt{3120} \cdot 13 \\
\sqrt{1022} \cdot 14+\sqrt{2720} \cdot 23 \\
\sqrt{1533} \cdot 15+\sqrt{1460} \cdot 24 \\
\sqrt{2574} \cdot 16+\sqrt{2244} \cdot 25)\end{array}$ & $\begin{array}{l}2<3<5<6<7<8< \\
9<10\end{array}$ & 1 & $\mathrm{E}$ & 1 & 4 & 4 \\
\hline 38 & $\begin{array}{l}(0,0, \sqrt{10925} \cdot 12, \sqrt{16709} .13 \\
\sqrt{5642} \cdot 23, \sqrt{14030} .14 \\
\sqrt{11718} \cdot 25, \sqrt{9690} .26+ \\
\sqrt{12444} .34)\end{array}$ & $\begin{array}{l}65<82<147<212< \\
229<277<311< \\
359\end{array}$ & 1 & $\mathrm{E}$ & 2 & 4 & 5 \\
\hline 39 & $\begin{array}{l}\left(0,0, \sqrt{\frac{495}{2716}} \cdot 12, \sqrt{\frac{55}{201}} \cdot 13, \sqrt{\frac{58}{497}} \cdot 23,\right. \\
\sqrt{\frac{699}{2770}} \cdot 14, \sqrt{\frac{31}{497}} \cdot 16+ \\
\left.\sqrt{\frac{50}{251}} \cdot 25, \sqrt{\frac{77}{488}} \cdot 26+\sqrt{\frac{220}{1007}} \cdot 34\right)\end{array}$ & $\begin{aligned} 1478229562< \\
2217119646< \\
3695349208,< \\
5173384043< \\
5912403141< \\
6651461046< \\
8129676552< \\
868681594\end{aligned}$ & 1 & $\mathrm{E}$ & 1 & 2 & 5 \\
\hline
\end{tabular}




\begin{tabular}{|c|c|c|c|c|c|c|c|}
\hline 40 & $\begin{array}{l}(0,0,2 \sqrt{3} \cdot 12,4 \cdot 13,0,2 \sqrt{3} \cdot 14,2 \cdot 15 \\
\sqrt{13} \cdot 26+\sqrt{13} \cdot 34)\end{array}$ & $\begin{array}{l}8<27<35<43<48< \\
51<56<78\end{array}$ & 1 & $\mathrm{E}$ & 3 & 2 & 5 \\
\hline 41 & $\begin{array}{l}(0,0,2 \cdot 12, \sqrt{5} .13,0,2 \cdot 14,1.15+ \\
1.23,2.26+2.34)\end{array}$ & $\begin{array}{l}3<8<11<14<16< \\
17<19<25\end{array}$ & 1 & $\mathrm{E}$ & 2 & 2 & 5 \\
\hline 42 & $\begin{array}{l}(0,0,6 \sqrt{2} \cdot 12, \sqrt{85} \cdot 13,0,8 \cdot 14+ \\
\sqrt{34} \cdot 23, \sqrt{34} \cdot 15,9 \cdot 26+6 \sqrt{2} \cdot 34)\end{array}$ & $\begin{array}{l}4<8<12<16<17< \\
20<21<28\end{array}$ & 1 & $\mathrm{E}$ & 2 & 2 & 5 \\
\hline 43 & $\begin{array}{l}(0,0, \sqrt{11} \cdot 12, \sqrt{21} \cdot 13, \sqrt{21} \cdot 14 \\
3 \sqrt{3} \cdot 23,2 \sqrt{5} \cdot 15, \sqrt{14} \cdot 16+ \\
3 \sqrt{2} .24)\end{array}$ & $\begin{array}{l}19<50<69<88< \\
107<119<126< \\
138\end{array}$ & 1 & $\mathrm{E}$ & 2 & 4 & 5 \\
\hline 44 & $\begin{array}{l}(0,0,8 \cdot 12, \sqrt{85} \cdot 13,6 \sqrt{2} \cdot 14, \sqrt{34} \cdot 15, \\
+9.34)\end{array}$ & $\begin{array}{l}2<11<13<15< \\
17<19<21<28\end{array}$ & 1 & $\mathrm{E}$ & 2 & 2 & 6 \\
\hline 45 & $\begin{array}{l}(0,0, \sqrt{26} \cdot 12, \sqrt{31} \cdot 13, \sqrt{30} \cdot 14 \\
4.15, \sqrt{11} \cdot 16+\sqrt{11} \cdot 23, \sqrt{26} \cdot 25+ \\
\sqrt{30} \cdot 34)\end{array}$ & $\begin{array}{l}1<4<5<6<7<8< \\
9<11\end{array}$ & 1 & $\mathrm{E}$ & 1 & 2 & 6 \\
\hline 46 & $\begin{array}{l}(0,0, \sqrt{603} \cdot 12, \sqrt{1995} \cdot 13 \\
\sqrt{1943} \cdot 14, \sqrt{2680} \cdot 15, \sqrt{1742} \cdot 16+ \\
\sqrt{2695} \cdot 23, \sqrt{1824} \cdot 17+ \\
\sqrt{2464} \cdot 24)\end{array}$ & $\begin{array}{l}1<4<5<6<7<8< \\
9<10\end{array}$ & 1 & $\mathrm{E}$ & 1 & 4 & 7 \\
\hline 47 & $\begin{array}{l}(0,0,0,0, \sqrt{19} \cdot 12, \sqrt{22} \cdot 23,2 \sqrt{3} \cdot 15+ \\
\sqrt{13} \cdot 24, \sqrt{19} \cdot 17+\sqrt{22} \cdot 36+ \\
\sqrt{13} .45)\end{array}$ & $\begin{array}{l}18<22<27<36< \\
40<49<58<76\end{array}$ & 1 & $\mathrm{E}$ & 2 & 2 & 4 \\
\hline 48 & $\begin{array}{l}(0,0,0,0, \sqrt{29} \cdot 12, \sqrt{22} \cdot 13, \sqrt{22} .15 \\
+\sqrt{29} \cdot 24, \sqrt{21} .17+4 \sqrt{2} .36+ \\
\sqrt{21} .45)\end{array}$ & $\begin{array}{l}11<\quad 20<\quad 21< \\
22<31<32<42<53\end{array}$ & 1 & $\mathrm{E}$ & 2 & 2 & 4 \\
\hline 49 & $\begin{array}{l}(0,0,0,0,3 \sqrt{2} \cdot 12,3 \sqrt{2} \cdot 13,3 \sqrt{2} \cdot 14+ \\
\sqrt{10} \cdot 23, \sqrt{21} \cdot 27+4 \cdot 36+ \\
\sqrt{21} \cdot 45)\end{array}$ & $\begin{array}{l}46<51<56<61< \\
97<102<107<158\end{array}$ & 1 & $\mathrm{E}$ & 2 & 2 & 4 \\
\hline 50 & $\begin{array}{l}(0,0,0,2 \sqrt{3} \cdot 12, \sqrt{21} \cdot 14, \sqrt{14} \cdot 23 \\
4.15,3.27+\sqrt{14} \cdot 36+2 \sqrt{3} \cdot 45)\end{array}$ & $\begin{array}{l}2<4<5<6<8<9< \\
10<14\end{array}$ & 1 & $\mathrm{E}$ & 2 & 2 & 5 \\
\hline 51 & $\begin{array}{l}(0,0,3.12,0,0, \sqrt{14} .13,3.16+ \\
\sqrt{6} .24,3.27+3.36+\sqrt{6} .45)\end{array}$ & $\begin{array}{l}5<9<14<15<18< \\
19<24<33\end{array}$ & 1 & $\mathrm{E}$ & 2 & 2 & 5 \\
\hline 52 & $\begin{array}{l}(0,0,0,0, \sqrt{238} .14, \sqrt{253} .23, \\
\sqrt{253} .15+2 \sqrt{21} .34, \sqrt{153} .17+ \\
\sqrt{230} .26+3 \sqrt{6} .35)\end{array}$ & $\begin{array}{l}11<18<22<25< \\
36<40<47<58\end{array}$ & 1 & $\mathrm{E}$ & 2 & 2 & 4 \\
\hline 53 & $\begin{array}{l}(0,0,0,0,6 \sqrt{23} \cdot 13,5 \sqrt{30} .14+ \\
\sqrt{253} \cdot 23,5 \sqrt{39} \cdot 24,3 \sqrt{70} .17+ \\
3 \sqrt{91} \cdot 26+\sqrt{805} .35)\end{array}$ & $\begin{array}{l}83<90<97<104< \\
180<187<194< \\
277\end{array}$ & 1 & $\mathrm{E}$ & 2 & 2 & 3 \\
\hline 54 & $\begin{array}{l}(0,0,0,0,4 \sqrt{2} \cdot 13, \sqrt{21} \cdot 14, \sqrt{22} \cdot 15 \\
+\sqrt{29} .24, \sqrt{21} .17+\sqrt{29} .26+ \\
\sqrt{22} .35)\end{array}$ & $\begin{array}{l}21<40<42<44< \\
63<65<84<105\end{array}$ & 1 & $\mathrm{E}$ & 2 & 2 & 4 \\
\hline 55 & $\begin{array}{l}(0,0,0,0, \sqrt{414} .13, \sqrt{260} .14+ \\
\sqrt{391} .23, \sqrt{529} .15+\sqrt{338} .24 \\
\sqrt{430} .17+\sqrt{559} .26+\sqrt{138} .35)\end{array}$ & $\begin{array}{l}2<3<4<5<6<7< \\
8<10\end{array}$ & 1 & $\mathrm{E}$ & 1 & 2 & 4 \\
\hline 56 & $\begin{array}{l}(0,0,0,0,3.12,2 \sqrt{2} .15+ \\
2.23,2.25+2 \sqrt{2} .34,3.16+2.35)\end{array}$ & $\begin{array}{l}10<19<20<28< \\
29<39<48<49\end{array}$ & 1 & $\mathrm{E}$ & 2 & 2 & 4 \\
\hline
\end{tabular}




\begin{tabular}{|c|c|c|c|c|c|c|c|}
\hline 57 & $\begin{array}{l}(0,0,0,0, \sqrt{21} \cdot 12,2 \sqrt{5} \cdot 14+ \\
\sqrt{14} \cdot 23, \sqrt{21} \cdot 25+ \\
\sqrt{11} \cdot 34,3 \cdot \sqrt{3} \cdot 16+3 \sqrt{2} \cdot 35)\end{array}$ & $\begin{array}{l}38<50<63<75< \\
88<113<138<151\end{array}$ & 1 & $\mathrm{E}$ & 2 & 2 & 3 \\
\hline 58 & $\begin{array}{l}(0,0,0,0, \sqrt{2} \cdot 12, \sqrt{2} \cdot 14+ \\
\sqrt{2} .23, \sqrt{2} \cdot 24, \sqrt{3} \cdot 16+\sqrt{3} \cdot 35)\end{array}$ & $\begin{array}{l}5<6<7<8<11< \\
13<14<18\end{array}$ & 1 & $\mathrm{E}$ & 3 & 2 & 3 \\
\hline 59 & $\begin{array}{l}(0,0,0,0,2.12, \sqrt{3} .14+ \\
2.23, \sqrt{2} .15+\sqrt{3} .24, \sqrt{5} .16+ \\
\sqrt{5} .35)\end{array}$ & $\begin{array}{l}8<11<13<16< \\
19<24<27<32\end{array}$ & 1 & $\mathrm{E}$ & 2 & 2 & 3 \\
\hline 60 & $\begin{array}{l}(0,0,0,0,3 \sqrt{3} \cdot 12,3 \sqrt{3} \cdot 13, \sqrt{14} \cdot 16+ \\
4.25+\sqrt{14} \cdot 34, \sqrt{23} \cdot 26+ \\
\sqrt{23} \cdot 35)\end{array}$ & $\begin{array}{l}27<28<29<54< \\
55<56<83<84\end{array}$ & 1 & $\mathrm{E}$ & 2 & 2 & 3 \\
\hline 61 & $\begin{array}{l}(0,0,0, \sqrt{22} \cdot 12,3 \sqrt{2} \cdot 13, \sqrt{22} \cdot 14 \\
2 \sqrt{7} \cdot 24, \sqrt{11} \cdot 17+\sqrt{14} \cdot 26+ \\
2 \sqrt{7} .35)\end{array}$ & $\begin{array}{l}4<5<7<9<11< \\
13<14<18\end{array}$ & 1 & $\mathrm{E}$ & 2 & 2 & 4 \\
\hline 62 & $\begin{array}{l}(0,0,0, \sqrt{13} \cdot 12,3 \cdot 13,4 \cdot 14,3.16+ \\
4.24,2 \sqrt{2} .17+2 \sqrt{2} \cdot 26+\sqrt{19} \cdot 35)\end{array}$ & $\begin{array}{l}2<4<5<6<7<8< \\
10<12\end{array}$ & 1 & $\mathrm{E}$ & 1 & 2 & 5 \\
\hline 63 & $\begin{array}{l}\quad(0,0,0, \sqrt{391} \cdot 12, \sqrt{414} \cdot 13 \\
\sqrt{430} \cdot 14, \sqrt{138} .15+ \\
\sqrt{559} \cdot 24, \sqrt{260} .17+\sqrt{338} \cdot 26+ \\
\sqrt{529} .35)\end{array}$ & $\begin{array}{l}2<3<4<5<6<7< \\
8<10\end{array}$ & 1 & $\mathrm{E}$ & 1 & 2 & 4 \\
\hline 64 & $\begin{array}{l}(0,0,3 \cdot 12,0, \sqrt{21} \cdot 23, \sqrt{14} \cdot 14 \\
\sqrt{14} \cdot 16+2 \sqrt{3} \cdot 25,2 \sqrt{3} \cdot 17+4.35)\end{array}$ & $\begin{array}{l}2<3<5<7<8<9< \\
11<13\end{array}$ & 1 & $\mathrm{E}$ & 2 & 4 & 5 \\
\hline 65 & $\begin{array}{l}(0,0,2 \sqrt{3} \cdot 12,0,2 \sqrt{5} \cdot 13, \sqrt{15} \cdot 15 \\
2 \sqrt{3} \cdot 24,2 \sqrt{3} \cdot 26+\sqrt{15} \cdot 35)\end{array}$ & $\begin{array}{l}13<24<37<48< \\
50<63<72 ;<87\end{array}$ & 1 & $E$ & 3 & 4 & 5 \\
\hline 66 & $\begin{array}{l}(0,0,2 \sqrt{6} \cdot 12,0,2 \sqrt{10} \cdot 13, \sqrt{31} .15 \\
2.16+2 \sqrt{6} .24,2 \sqrt{6} .26+\sqrt{31} \cdot 35)\end{array}$ & $\begin{array}{l}25<52<77<100< \\
102<127<152< \\
179\end{array}$ & 1 & $\mathrm{E}$ & 2 & 2 & 5 \\
\hline 67 & $\begin{array}{l}(0,0,2 \sqrt{6} \cdot 12,0,2 \sqrt{10} \cdot 13,2 \cdot 14, \\
2 \sqrt{6} \cdot 15+2 \sqrt{6} \cdot 24, \sqrt{31} \cdot 27+ \\
\sqrt{31} \cdot 35)\end{array}$ & $\begin{array}{l}32<45<77<96< \\
109<128<141< \\
186\end{array}$ & 1 & $\mathrm{E}$ & 2 & 2 & 5 \\
\hline 68 & $\begin{array}{lll}(0,0,0,0, \quad 1.12, & 1.13, & 1.15+1.24 \\
1.16+1.25+1.34) & & \end{array}$ & $\begin{array}{l}7<10<13<14< \\
17<20<24<27\end{array}$ & 0 & - & 2 & 2 & 3 \\
\hline 69 & $(0,0,0,12,13,14,15+23,16+24)$ & $\begin{array}{l}5<10<14<15< \\
19<20<24<25\end{array}$ & 0 & - & 2 & 4 & 4 \\
\hline 70 & $\begin{array}{l}(0,0,0, \sqrt{2} \cdot 12, \sqrt{7} \cdot 13,3 \cdot 23,2 \sqrt{2} \cdot 14 \\
\sqrt{7} \cdot 16+3 \cdot 25)\end{array}$ & $\begin{array}{l}7<9<11<16<18 \\
<20<23<27\end{array}$ & 1 & $\mathrm{E}$ & 3 & 4 & 3 \\
\hline 71 & $\begin{array}{l}(0,0,0,0, \sqrt{30} \cdot 13, \sqrt{22} \cdot 16+ \\
5.23, \sqrt{22} \cdot 24,6 \cdot 16+\sqrt{30} \cdot 25)\end{array}$ & $(5,6,8,9,13,14,15,19$ & 1 & $\mathrm{E}$ & 3 & 4 & 3 \\
\hline 72 & $\begin{array}{l}\left(0,0, \sqrt{\frac{19}{269}} \cdot 12, \sqrt{\frac{9590}{46357}} \cdot 13, \sqrt{\frac{11900}{46357}} \cdot 2\right. \\
\sqrt{\frac{36}{151}} \cdot 14, \sqrt{\frac{13289}{139071}} \cdot 15+ \\
\sqrt{\frac{16490}{139071}} \cdot 24, \sqrt{34} \cdot 5+\sqrt{\frac{22}{151}} \cdot 16+ \\
\left.\sqrt{\frac{86}{453}} \cdot 25\right)\end{array}$ & $\begin{array}{l}29588<44383 \\
<73971 \\
<103562 \\
<118357 \\
<133152 \\
<147947 \\
<162742\end{array}$ & 1 & $\mathrm{E}$ & 1 & 4 & 5 \\
\hline
\end{tabular}




\begin{tabular}{|l|l|l|l|l|l|l|l|}
\hline 73 & $(0,0,0, \sqrt{7} \cdot 12, \sqrt{7} \cdot 13, \sqrt{2} \cdot 24, \sqrt{2} \cdot 25$ & $5<8<10<13$ & 2 & $\mathrm{E}$ & 3 & 4 & 3 \\
& $<\sqrt{2} \cdot 34+3.45)$ & $<23<28$ & & & & \\
\end{tabular}

Table 2. 8-dimensional nilsoliton metric Lie algebras-nullity 2

\begin{tabular}{|c|c|c|c|c|c|c|c|}
\hline & Lie Bracket & $\begin{array}{l}\text { Derivation } \\
\text { Type }\end{array}$ & $|\mathbf{A o M}|$ & $\begin{array}{l}\text { E/ } \\
\text { VPA }\end{array}$ & Rank & Index & NI \\
\hline 1 & $\begin{array}{l}(0,0,0, \sqrt{10} .13, \sqrt{11} .14, \sqrt{5} .15+ \\
\sqrt{10} .23, \sqrt{11} .16+\sqrt{11} .24 \\
\sqrt{10} .17+\sqrt{10} .25)\end{array}$ & $\begin{array}{l}8<24<35< \\
43<51< \\
59<67<75\end{array}$ & 2 & $\mathrm{E}$ & 2 & 4 & 6 \\
\hline 2 & $\begin{array}{l}(0,0,0,1 \cdot 13,1 \cdot 14,1.15+1.23,1 \cdot 16+ \\
1.24,1.17+1.25+1.34) \\
\end{array}$ & $\begin{array}{l}1<3<4< \\
5<6<7< \\
8<9\end{array}$ & 2 & VPA & 1 & 2 & 6 \\
\hline 3 & $\begin{array}{l}(0,0,0,1 \cdot 12,1.13,1.23,1.15+ \\
1.24,1 \cdot 16+1.25+1.34)\end{array}$ & $\begin{array}{l}5<6<7< \\
11<12< \\
13<17<18\end{array}$ & 1 & VPA & 2 & 4 & 3 \\
\hline 4 & $\begin{array}{l}(0,0,1.12,1.13,1.23,1.15+1.24,1.25 \\
1.16+1.34)\end{array}$ & $\begin{array}{l}8<9<17< \\
25<26< \\
34<35<42\end{array}$ & 2 & VPA & 2 & 2 & 5 \\
\hline 5 & $\begin{array}{l}(0,0,1 \cdot 12,1 \cdot 13,0,1 \cdot 15+1 \cdot 23,1 \cdot 16+ \\
1 \cdot 24,1 \cdot 17+1 \cdot 34)\end{array}$ & $\begin{array}{l}5<14<19< \\
24<28< \\
33<38<43\end{array}$ & 2 & EA & 2 & 4 & 5 \\
\hline 6 & $\begin{array}{l}(0,0,1 \cdot 12,1 \cdot 13,1 \cdot 14,1 \cdot 15,1 \cdot 25+ \\
1 \cdot 34,1 \cdot 17+1.26)\end{array}$ & $\begin{array}{l}1<4<5< \\
6<7<8< \\
11<12\end{array}$ & 2 & VPA & 2 & 2 & 6 \\
\hline 7 & $\begin{array}{l}(0,0,1.12,1.13,1.14,1.15+1.23,1.25+ \\
1.34,1.17+1.26)\end{array}$ & $\begin{array}{l}1<3<4<5 \\
<6<7<9 \\
<10\end{array}$ & 2 & VPA & 1 & 2 & 6 \\
\hline 8 & $\begin{array}{l}(0,0, \sqrt{806361} \cdot 12,120 \sqrt{78} \cdot 13 \\
\sqrt{922743} \cdot 14,4 \sqrt{29666} \cdot 15+ \\
20 \sqrt{1430} \cdot 23,12 \sqrt{4914} \cdot 16+ \\
6 \sqrt{10010} \cdot 24, \sqrt{837983} \cdot 25+ \\
\sqrt{958929} \cdot 34)\end{array}$ & $\begin{array}{l}1<3<4<5 \\
<6<7<8 \\
<9\end{array}$ & 2 & VPA & 1 & 4 & 6 \\
\hline 9 & $\begin{array}{l}(0,0,0, \sqrt{14} \cdot 12, \quad \sqrt{14} \cdot 13, \quad \sqrt{6} \cdot 24 \\
\sqrt{13} \cdot 25+\sqrt{13} \cdot 34, \sqrt{14} \cdot 17+\sqrt{13} .45)\end{array}$ & $\begin{array}{l}5<8<10 \\
<13<15 \\
<21<23 \\
<28\end{array}$ & 2 & $E$ & 3 & 2 & 4 \\
\hline 10 & $\begin{array}{l}(0,0,0,1 \cdot 12,1 \cdot 13,1 \cdot 24,1 \cdot 25+ \\
1.34,1 \cdot 17+1.26+1.45)\end{array}$ & $\begin{array}{l}20<29< \\
38<49< \\
58<78< \\
87<107\end{array}$ & 2 & VPA & 2 & 2 & 4 \\
\hline 11 & $\begin{array}{l}(0,0,0,1 \cdot 12,1 \cdot 14+1.23,0,1 \cdot 15+ \\
1.34,1 \cdot 27+1.36+1.45)\end{array}$ & $\begin{array}{l}22<25< \\
44<47< \\
69<72< \\
91<116\end{array}$ & 2 & VPA & 1 & 4 & 5 \\
\hline 12 & $\begin{array}{l}(0,0,0,1.13,0,1.14+1.23,1.16+ \\
1.24,1.17+1.26+1.35)\end{array}$ & $\begin{array}{l}7<14<17 \\
<24<28 \\
<31<38 \\
<45\end{array}$ & 2 & VPA & 2 & 4 & 5 \\
\hline
\end{tabular}




\begin{tabular}{|c|c|c|c|c|c|c|c|}
\hline 13 & $\begin{array}{l}(0,0,0,1 \cdot 13,1 \cdot 23,1 \cdot 14,1 \cdot 15+ \\
1 \cdot 24,1 \cdot 17+1 \cdot 26+1 \cdot 35)\end{array}$ & $\begin{array}{l}8<13<16< \\
24<29< \\
32<37<45\end{array}$ & 2 & VPA & 2 & 4 & 4 \\
\hline 14 & $\begin{array}{l}(0,0,0,1 \cdot 12,1 \cdot 14+1.23,1.24,1 \cdot 15+ \\
1.34,1 \cdot 17+1.26+1.35)\end{array}$ & $\begin{array}{l}18914< \\
28370< \\
37828< \\
47284<66199 \\
<75657< \\
85113< \\
104027\end{array}$ & 2 & VPA & 1 & 2 & 5 \\
\hline 15 & $\begin{array}{l}(0,0,0,1.12,1.13,1.14+1.23,1.15+ \\
1.24,1.17+1.26+1.35)\end{array}$ & $\begin{array}{l}2<3<4<5 \\
<6<7<8 \\
<10\end{array}$ & 1 & VPA & 1 & 2 & 4 \\
\hline 16 & $\begin{array}{r}(0,0,1.12,0,1.23,1.24,1 \cdot 16+1.25 \\
+1.34,1.17+1.35)\end{array}$ & $\begin{array}{l}7<8<15< \\
16<23< \\
24<31<38\end{array}$ & 2 & VPA & 2 & 2 & 5 \\
\hline 17 & $\begin{aligned}(0,0,1 \cdot 12,0,1 \cdot 14 & +1.23,0,1 \cdot 16 \\
& +1.25+1.34,1 \cdot 17 \\
& +1.35)\end{aligned}$ & $\begin{array}{l}7<8<15< \\
16<23< \\
24<31<38 \\
\end{array}$ & 2 & VPA & 2 & 2 & 5 \\
\hline 18 & $\begin{array}{l}(0,0, \sqrt{37} \cdot 12,0,3 \sqrt{5} \cdot 13, \sqrt{37} \cdot 15+ \\
\sqrt{37} \cdot 24,5 \cdot 16+5 \cdot 34,2 \sqrt{10} \cdot 26+ \\
2 \sqrt{10} \cdot 35)\end{array}$ & $\begin{array}{l}41<71< \\
112<123< \\
153<194< \\
235<265\end{array}$ & 2 & $\mathrm{E}$ & 2 & 2 & 5 \\
\hline 19 & $\begin{array}{l}(0,0,3 \sqrt{2} \cdot 12,4 \cdot 13, \sqrt{19} \cdot 14,0,3 \sqrt{2} \cdot 25 \\
+\sqrt{19} \cdot 34, \sqrt{14} \cdot 17+2 \cdot 26+ \\
\sqrt{14} \cdot 35)\end{array}$ & $\begin{array}{l}1<4<5< \\
6<7<8< \\
11<12\end{array}$ & 2 & $\mathrm{E}$ & 2 & 2 & 6 \\
\hline 20 & $\begin{array}{l}(0,0,1 \cdot 12,1 \cdot 13,1 \cdot 14,1 \cdot 23,1 \cdot 25+ \\
1.34,1 \cdot 17+1.35)\end{array}$ & $\begin{array}{l}1<4<5< \\
6<7<9< \\
11<12\end{array}$ & 2 & VPA & 2 & 2 & 6 \\
\hline
\end{tabular}

Table 3. 8-dimensional nilsoliton metric Lie algebras-nullity3

\begin{tabular}{|c|c|c|c|c|c|c|c|}
\hline & Lie Bracket & $\begin{array}{l}\text { Derivation } \\
\text { Type }\end{array}$ & $|\mathbf{A o M}|$ & E/VPA & Rank & Index & NI \\
\hline 1 & $\begin{array}{l}(0,0,0, \sqrt{5} .13, \sqrt{5} .14+\sqrt{5} .23, \sqrt{6} .15+ \\
\sqrt{6} .24, \sqrt{5} .16+\sqrt{6} .25, \sqrt{5} .17+\sqrt{5} .26)\end{array}$ & $\begin{array}{l}1<2< \\
5<6< \\
7<8< \\
9<10\end{array}$ & 3 & $E$ & 2 & 4 & 6 \\
\hline 2 & $\begin{array}{l}(0,0,0,1.13,1.14+1.23,1.15+ \\
1.24,1.16+1.25,1.17+1.26+1.34)\end{array}$ & $\begin{array}{l}1<2< \\
4<5< \\
6<7< \\
8<9\end{array}$ & 3 & VPA & 1 & 2 & 6 \\
\hline 3 & $\begin{array}{l}(0,0,0, \sqrt{15} \cdot 12, \sqrt{14} \cdot 14+ \\
\sqrt{14} .23, \sqrt{15} .15+\sqrt{14} \cdot 34, \sqrt{10} \cdot 16+ \\
\sqrt{10} \cdot 35, \sqrt{15} .26+\sqrt{15} .45)\end{array}$ & $\begin{array}{l}8<13< \\
16<21< \\
29<37< \\
45<50\end{array}$ & 3 & E & 2 & 2 & 5 \\
\hline 4 & $\begin{array}{l}(0,0, \sqrt{15} \cdot 12, \sqrt{14} \cdot 13, \sqrt{15} .14, \sqrt{15} .25+ \\
\sqrt{15} .34, \sqrt{14} .16+\sqrt{14} .35, \sqrt{10} .17+ \\
\sqrt{10} .45)\end{array}$ & $\begin{array}{l}1<20< \\
21<22< \\
23<43< \\
44<45\end{array}$ & 3 & E & 2 & 2 & 7 \\
\hline
\end{tabular}




\begin{tabular}{|c|c|c|c|c|c|c|c|}
\hline 5 & $\begin{array}{l}(0,0,2 \sqrt{14} .12,2 \sqrt{6} .13, \sqrt{56} .14,2 \sqrt{14} .25+ \\
\sqrt{57} .34,2 \sqrt{13} .16+2 \sqrt{13} .35,2 \sqrt{14} .27+ \\
2 \sqrt{13} .36)\end{array}$ & $\begin{array}{l}13<20< \\
33<46< \\
59<79< \\
92<112\end{array}$ & 3 & $E$ & 2 & 2 & 7 \\
\hline 6 & $\begin{array}{l}(0,0,0 \\
\sqrt{323} \cdot 12,2 \sqrt{17} \cdot 14 \\
+4 \sqrt{7} \cdot 23, \sqrt{252} \cdot 24, \sqrt{323} \cdot 15 \\
+4 \sqrt{7} \cdot 34,6 \sqrt{5} \cdot 27,+8 \sqrt{5} \cdot 36+6 \sqrt{5} \cdot 45)\end{array}$ & $\begin{array}{l}22<25< \\
44<47< \\
69<72< \\
91<116\end{array}$ & 2 & E & 2 & 2 & 5 \\
\hline 7 & $\begin{aligned}(0,0,1.12,0,1.13,1.14+1.23,1.16 \\
+1.25,1.27+1.36+1.45)\end{aligned}$ & $\begin{array}{l}4<5<9< \\
10<13< \\
14<18<23 \\
\end{array}$ & 2 & VPA & 2 & 2 & 5 \\
\hline 8 & $\begin{aligned}(0,0,1.12,1.13, & 1.14,1.15+1.23,1.16 \\
& +1.24,1.27+1.36+1.45)\end{aligned}$ & $\begin{array}{l}1<3<4< \\
5<6<7< \\
8<11\end{array}$ & 2 & VPA & 1 & 2 & 7 \\
\hline 9 & $\begin{aligned}(0,0,6 \sqrt{5} .12,0,4 \sqrt{7} \cdot 14+4 \sqrt{17} .23 \\
6 \sqrt{7} .24,8 \sqrt{5} .16+6 \sqrt{5} .25 \\
+4 \sqrt{7} .34, \sqrt{323} .35)\end{aligned}$ & $\begin{array}{l}7<8<15< \\
16<23< \\
24<31<38\end{array}$ & 2 & $\mathrm{E}$ & 2 & 2 & 5 \\
\hline 10 & $\begin{aligned}(0,0,6 \sqrt{5} .12,4 & \sqrt{17} .13, \sqrt{323} .14 \\
& 6 \sqrt{7} \cdot 15,6 \sqrt{5} \cdot 25 \\
& +\sqrt{323} \cdot 34,8 \sqrt{5} .26 \\
& +4 \sqrt{7} \cdot 35)\end{aligned}$ & $\begin{array}{l}1<4<5< \\
6<7<8< \\
11<12\end{array}$ & 2 & $E$ & 2 & 2 & 6 \\
\hline 11 & $\begin{aligned} &(0,0,2 \sqrt{2} .12, \sqrt{5} .13, \sqrt{7} .14+\sqrt{5} .23,1.15 \\
&+ 1.24,2 \sqrt{2} .25 \\
&+\sqrt{7} .34, \sqrt{7} .17+\sqrt{7} .35)\end{aligned}$ & $\begin{array}{l}1<2<3< \\
4<5<6< \\
7<8\end{array}$ & 3 & $\mathrm{E}$ & 1 & 2 & 6 \\
\hline
\end{tabular}

\section{CONFLICTS OF INTEREST}

No conflict of interest was declared by the author.

\section{REFERENCES}

[1] Beck, R., Kolman, B., Stewant, I., "Computing the structure of a Lie algebra", book chapter in Nonassociative rings and algebras, $1^{\text {st }}$ ed, Editor(s): Beck, R., Kolman, B., New York, Academic Press, (1977).

[2] Ceballos, M., Nez, J., Tenorio, F., "Algorithm to compute minimal matrix representation of nilpotent Lie algebras", International Journal of Computer Mathematics, 97(1-2): 275-293, (2020).

[3] De Graaf, W.A., Lie algebras theory and algorithms $1^{\text {st }}$ ed, Elsevier, Amsterdam, (2000).

[4] De Graaf, W.A. "Calculating the structure of semi-simple Lie algebra", Journal of Pure and Applied Algebra, (117 \& 118): 319-329, (1997).

[5] Ronyai, L., "Computing the structure of finite algebra", Journal of Symbolic Computation, 9: 355$373,(1990)$.

[6] Nikolayevsky, Y., "Einstein solvmanifolds with a simple Einstein derivation", Geometriae Dedicata, 135: 87-102, (2008). 
[7] Lauret, J., "Einstein solvmanifolds and nilsolitons", Contemporary Mathematics, 491: 1-35, (2009).

[8] Lauret, J., Will, C., "Einstein solvmanifolds: existence and nonexistence questions", Mathematische Annalen, 350(1): 199-225, (2011).

[9] Arroyo, R.M., "Filiform nilsolitons of dimension 8", Rocky Mountain Journal of Mathematics, 41(4): 1025-1043, (2011).

[10] Culma, E.A.F., "Classification of 7-dimensional Einstein nilradicals", Transformation Groups, 17(3): 639-656, (2012). DOI: 10.1007/s00031-012-9186-5

[11] De Graaf, W.A., "Classification of 6-dimensional nilpotent Lie algebras over fields of characteristic no 2", Journal of Algebra, 309(2): 640-653, (2007).

[12] Lauret, J., "Finding Einstein solvmanifolds by a variational method", Mathematische Zeitschrift, 241(1): 83-99, (2002).

[13] Payne, T.L., "Geometric invariants for nilpotent metric Lie algebras with applications to moduli spaces of nilsoliton metrics", Annals of Global Analysis and Geometry, 41(2): 139-160, (2012).

[14] Will, C., "Rank-one Einstein solvmanifolds of dimension 7", Differential Geometry and its Applications, 19(3): 307-318, (2003).

[15] Kadioglu, H., Payne, T.L., "Computational methods for nilsoliton metric Lie algebras I", Journal of Symbolic Computation, 50: 350 -373, (2013).

[16] Culma, E.A.F., "Classification of 7-dimensional Einstein nilradicals II", Technical Report, arXiv: 1105.4493 (2011).

[17] Kadioglu, H., "Classification of ordered type soliton metric Lie algebras by a computational approach”, Abstract and Applied Analysis, Article ID 871930, (2013).

[18] Nikolayevsky, Y., "Einstein solvmanifolds and the pre-Einstein derivation", Transactions of the American Mathematical Society, 363(8): 3935-3958, (2011).

[19] Kadioglu, H., "A computational procedure on higher dimensional nilsolitons", Mathematical Methods in the Applied Sciences, 42(16): 5390-5397, (2019).

[20] Kadioglu, H., "On some structural components of nilsolitons", Mathematical Problems in Engineering, Article ID 5540584, (2021).

[21] Payne, T.L., "The existence of soliton metrics for nilpotent Lie groups", Geometriae Dedicata, 145: 71-88, (2012).

[22] Adimi, H., Makhlouf, A., "Computing the index of Lie algebras", Proceedings of the Estonian Academy of Sciences, 59(4): 265-271, (2010). 